\title{
2815. A novel flexible planar loudspeaker with coils on polyimide diaphragm
}

\author{
Jium Ming Lin ${ }^{1}$, Cheng Hung Lin ${ }^{2}$ \\ ${ }^{1}$ Department of Electronic Engineering, Chung-Hua University, Hsin-Chu, 30012, Taiwan \\ ${ }^{2}$ College of Engineering, Chung-Hua University, Hsin-Chu, 30012, Taiwan \\ ${ }^{1}$ Corresponding author \\ E-mail:1jmlin@chu.edu.tw, ${ }^{2}$ b09306014@chu.edu.tw
}

Received 1 August 2017; received in revised form 19 October 2017; accepted 28 October 2017 DOI https://doi.org/10.21595/jve.2017.18923

Check for updates

Abstract. This paper presented a low cost flexible electro-dynamic planar loudspeaker (FEPL) with coils on polyimide diaphragm. The structure was very simple to put a flexible thin film diaphragm (electroplated traces of copper coil) above a flexible magnetic (placed in the bottom of cavity), thus forming an integration of electromagnetic actuation and planar flexible structure. The advantages are as follows: (1) the new loudspeaker became a flat flexible one, which could not only directly drive the vibration film to increase the power efficiency, but reduce the total harmonic distortion (THD). (2) It could be used as flexible electronics and easily deployed on the surface of any object with magnetic property or using adhesive transfer tapes. To optimize the performance of sound pressure level (SPLs), this study made detailed analyses on the thickness of cavity, turns of coil, magnet polarization, and diaphragm dimension. Noted as the cavity height increasing, the magnetic flux densities of both vertically and horizontally polarized magnet were decreased. But they were nearly constant over the surface with the same height for the vertically polarized magnet. On the other hand, as the cavity height of the horizontally polarized magnet increased, the magnetic flux densities would be decreased rapidly at the locations moving farther away from the center point. Several kinds of planar-type loudspeakers for the coils wounded in spiral-in, spiral-in and spiral-out, and zigzag shapes and plated with copper and aluminum were applied for performance tests, and the comparisons with the traditional cone-type loudspeaker were also made. Finally, the sound pressure levels (SPLs) were compared for three rectangular coils with different dimensions and number of turns. These effects were not discussed in previous literature of study.

Keywords: flexible substrate, planar loudspeaker, sound pressure level.

\section{Introduction}

Flexible electronics has been a hot research and development topic in the electronics industry since the past few years. The reason is owing to the rapid growth of flexible electronic technology $[1,2]$. The speaker is an important part of the consumer electronics and has received many attentions for a hundred years [3-11]. Some progresses have been made to develop the flexible loudspeaker, and most of them have failed to commercialize. The method of electro-acoustic transduction could have applied many kinds of actuation mechanisms in loudspeaker design, such as electromagnetic [4, 12-16], piezoelectric [5-6], electrostatic [8, 17], and electro-thermal [18, 19] actuation methods. Among them the flexible and transparent loudspeakers with piezoelectric actuator were developed $[3,5]$ using the piezoelectric polymer PVDF. Their study showed that the PVDF driven flexible loudspeaker could produce $70 \mathrm{~dB}$ and $80 \mathrm{~dB}$ sound pressure levels (SPLs) in the frequency ranges of $1-20 \mathrm{kHz}$ and $400 \mathrm{~Hz}-10 \mathrm{kHz}$, respectively. However, the complex production process makes PVDF material be very expensive [6]. The frequency response of which is better in the higher one but not in the lower band. In 2009 Industrial Technology Research Institute (ITRI) at Taiwan filed a patent [17] of an ultrathin flexible loudspeaker using electrostatic actuator, it could produce sound in the ranges of medium to high frequencies such as $200 \mathrm{~Hz}-20 \mathrm{kHz}$ [11]. They combined arrays of tiny, bendable capacitance type speakers to produce a sound system by inkjet printing polymer electret on paper or plastic substrate, and then 
sandwiches this substrate between a pair of thin metal layers. Nevertheless, the drawbacks are that it can operate only at higher frequencies and voltages. Furthermore, other capacitance-based flexible loudspeakers, developed by Fujifilm [8], Yamaha Corporation [9] and Warwick audio technologies [10], can produce sound only in a specified direction and suffered the safety problems of higher operating voltages. Next, comes the inspired work of Xiao et al. applying the electro-thermal mechanism [19], they brought a light, flexible and stretchable loudspeaker using carbon nano-tubes (CNT). But this loudspeaker has a major drawback to develop an available method to make the thin films of CNTs. Thus, at this moment nobody has applied the electromagnetic actuation mechanism to make a flat flexible loudspeaker. But this paper presents a method to realize it, and shows that the new flat flexible loudspeaker can be used in pop up banners, portable exhibition stands, and other uses required.

This study has explored the possibility to develop a flexible electro-dynamic planar loudspeaker with coils on polyimide diaphragm. In the past, electromagnetic actuation has been proven to be the most efficient way to generate sound [4-13]. The proposed design adopts the structure of a single or double ended planar electro-dynamic loudspeaker [7, 14]. Fig. 1 showed the explosive structure of the proposed design. The key technologies were as follows: (1) To electroplate a layer of copper on a diaphragm of polyimide film directly, when an audio current was applied to the coil to generate an alternative magnetic field, which can interact with the permanent magnet located at the bottom of cavity, thus the resulting repulsive and attraction forces could exert on the diaphragm and produce sound pressure. (2) To substitute the conventional rigid magnet with a flexible magnet made of mixture of polymer and neodymium $(\mathrm{NdFeB})$ or ferrite material. So, the new planar FEPL became a flat flexible one with direct drive mode to increase the power conversion efficiency. By the way, since the diaphragm was without any curved structure, it can reduce the total harmonic distortion (THD).

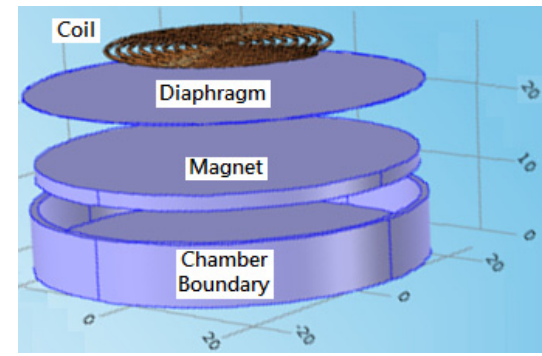

a)

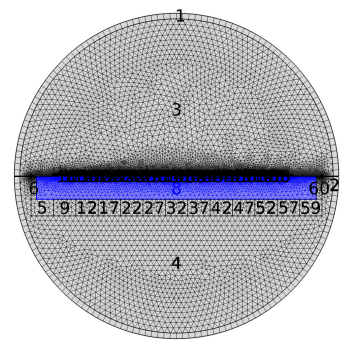

b)

Fig. 1. Proposed FEPL: a) explosive structure, b) side view of meshed geometry of loudspeaker

On the other hand, the force exerted to the traditional cone-type loudspeaker was operated in the manner of indirect mode as shown in Fig. 2, so the cone might be distorted at the conditions of larger amplitudes of vibrations, and thus both the performances of power conversion efficiency and the total harmonic distortion (THD) were not better than the newly proposed one. Besides, the thickness of the whole structure can be reduced by the new design, because the traditional rigid magnet was replaced by a flexible magnet made by the mixture of polymer and neodymium $(\mathrm{NdFeB})$ or ferrite material. The typical value of the magnetic flux density was $1.8 \mathrm{MgOe}$ (2730 gauss) or more [21-24].

This paper shows that the flexible loudspeaker is better when all the militating factors are considered later. Besides, a thin flexible film (polyimide, PI) is applied as the diaphragm electroplated with a copper coil. The 3D model of the flexible loudspeaker is applied using COMSOL; one can select the material properties of PI and permanent magnet of neodymium in the material libery and set the boundary conditions. The outer boundary of diaphragm is adhered on a fixed frame. The testing points of SPLs are at a height of $10 \mathrm{~cm}$ from the diaphragm center; and the magnetic fields are measured above the upper surface of speaker lying in the vertical 
planes along the center axes of the diaphragms. The monopole source model and the governing equations defined in COMSOL can be selected for application [23]. So, one can do all the simulations of Helmholtz equation for the acoustic pressure, Navier-Stokes equation for the stress and strain, acoustic-solid interaction and frequency domain analyses. Besides, the 2D triangles are applied as the shape of grids, which can generate the 3D tetrahedron, the numbers of the meshes at the terminals and boundaries are set as 204 and 505, respectively. Fig. 1(b) shows the meshed geometry of the baseline loudspeaker. In this paper the audio signal of $1 \mathrm{~V}$ was applied to the coil of loudspeaker, thus by Ampere's law and the COMSOL package, the generated AC magnetic field can interact with the permanent magnet at the bottom of cavity, and generates not only repulsive and attraction forces on the diaphragm, but the SPLs for measurement. This study made both mechano-acoustic and electrodynamic analyses on the thickness of cavity, diaphragm dimension, and magnet field configuration (respectively as vertically (axially) and horizontally (radically) polarized). Besides, this paper takes the SPLs of FEPL for three rectangular coils with different dimensions and number of turns into consideration. These effects are not discussed in previous literature of study. The paper is organized as follows: the first section is an introduction; the next part illustrates the FEPL structure configuration and optimization method; Section 3 is the performance tests and discussions for various shapes and materials of coils; the last part is a conclusion.

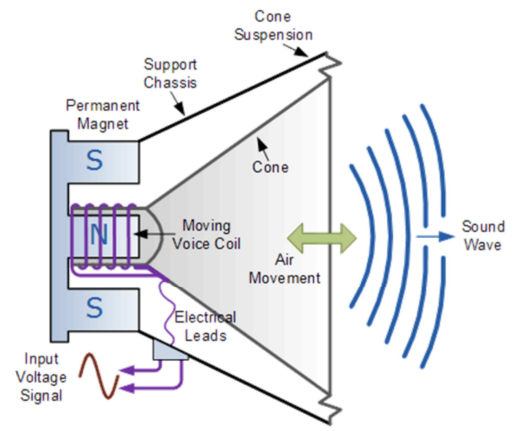

Fig. 2. Side view of traditional cone-type loudspeaker

\section{FEPL structure configuration and design}

The density, thermal coefficient of expansion and thickness of diaphragm (polyimide, endurable to $200{ }^{\circ} \mathrm{C}$ and light weight) are respectively as $1430 \mathrm{~kg} / \mathrm{m}^{3}, 5.5 \times 10^{-5} / \mathrm{K}$ and $0.122 \mathrm{~mm}$. The core structure was to substitute the previous rigid magnet with a flexible magnet made of mixture of polymer and neodymium $(\mathrm{NdFeB})$ or ferrite material. The typical value of magnetic flux density can be up to $1.8 \mathrm{MgOe}$ (2730 gauss) or more [21]. It is very good for flexible loudspeaker application. Besides, Fig. 1(a) shows that the copper coil is electroplated on the surface. Figs. 3(a) and 3(b) show the vertical and horizontal modes of magnet polarization, respectively. Since the loudspeaker under investigation is in a planar structure, two magnetic flux components are responsible for the vertical force actuation; the $x$-component $B_{x}$, and the $y$-component $B_{y}$. Due to different distributions of magnetic flux densities of $B_{x}$ and $B_{y}$, so the generated repulsive and attraction forces of the coils on the diaphragm are also different from each other.

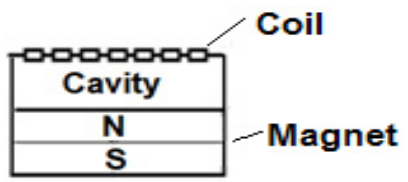

a) Vertical polarization of $B$

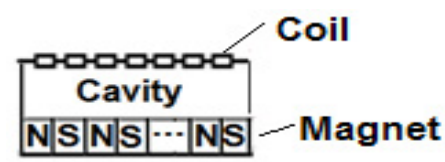

b) Horizontal polarization of B

Fig. 3. Two basic modes of magnet polarization 
Considering the structure of the proposed FEPL, the factors responsible for the performance are the FEPL cavity height between the distance of the coil and the magnet, the magnet polarization and the magnet arrangement. Let the diameter $(D)$ of the magnet be $3 \mathrm{~mm}$, and the cavity height is variable from $2 \mathrm{~mm}$ to $4 \mathrm{~mm}$. For easy of presentation, Figs. 4(a) and 4(b) show the magnitude curves of magnetic flux densities $B$ (in one dimension) for different cavity heights by using vertically and horizontally polarized magnets, respectively.
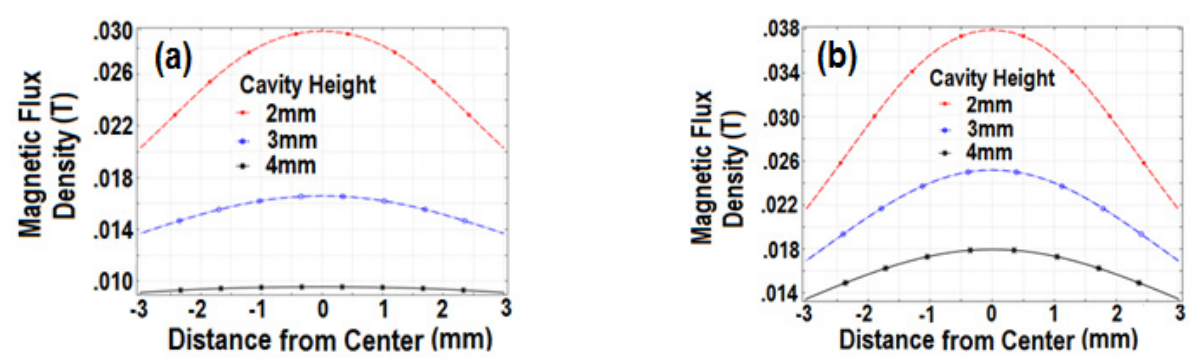

Fig. 4. Magnitude curves of $B$ above the speaker surface lying in the vertical planes along the center axes for different cavity height: a) vertically, b) horizontally polarized magnets (diameter $3 \mathrm{~mm}$ )

Note that if the cavity height increased, for either vertically or horizontally polarized magnet the magnetic flux density decreases rapidly away from the center point. Moreover, if the diameter of the magnet is enlarged to $6 \mathrm{~mm}$, Figs. 5(a) and 5(b) respectively show the magnetic flux densities by using the vertically and horizontally polarized magnets. Note that the magnitudes of the magnetic flux densities are larger than those shown in Figs. 4(a) and 4(b). Also note if the cavity height increases, the magnetic flux densities of both vertically and horizontally polarized magnet are decreased. But the distribution of the magnetic flux densities is still very uniform over the surface of the diaphragm at the same height of the vertically polarized magnet. However, if the cavity height of the horizontally polarized magnet increases, the magnetic flux densities will be decreased rapidly farther away from the center point.

Thus, one can predict the performance of speaker using the vertically polarized magnet is better than the horizontally polarized magnet. The reason is that the distribution of the magnetic flux density $B$ for the latter would not be so uniform, which will exert unequal forces on the coils, and many distortion modes of vibration can be easily excited.
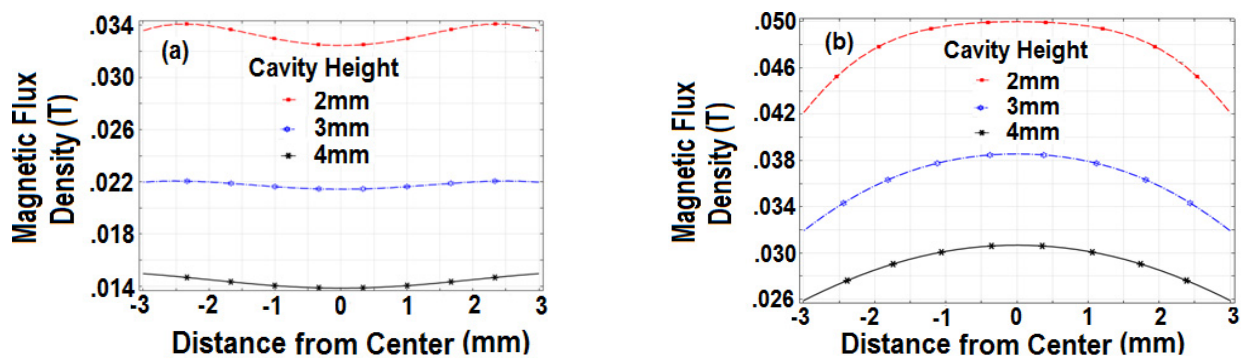

Fig. 5. Magnitude curves of $B$ for different cavity height by using: a) vertically,

b) horizontally polarized magnets (diameter $6 \mathrm{~mm}$ )

\section{Performance tests and discussions for various shapes and materials of coils}

In this section several kinds of planar-type loudspeakers $(B=0.3 \mathrm{~T})$ for the coils wounded in spiral-in, spiral-in and spiral-out, and zigzag shapes and plated with copper and aluminum are applied for performance tests, and the comparisons with the traditional cone-type loudspeaker are also made. By the way a typical audio signal (peak value be $3.3 \mathrm{~V}$ ) with frequencies from $20 \mathrm{~Hz}$ to $20 \mathrm{kHz}$ is applied for a period of 140 seconds to test the loudspeakers. 


\subsection{Copper and aluminum coils of spiral-in shape}

Firstly, two planar-type loudspeakers, with coils of spiral-in shape and plated with copper (resistance $5 \Omega$ ) and aluminum (resistance $300 \Omega$ ), are applied for test as shown in Fig. 6(a), the structure of the loudspeaker is also shown in Fig. 6(b). The performance tests and comparison with a traditional cone-type loudspeaker as shown in Fig. 7 are also made.
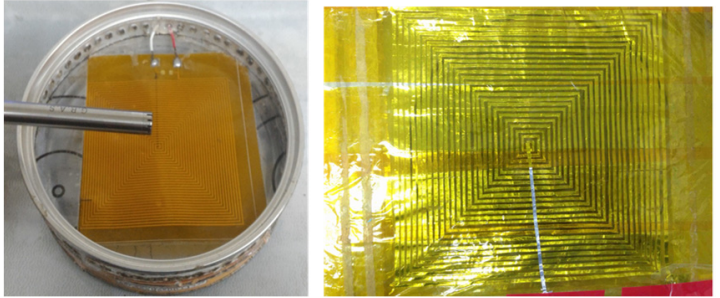

a)

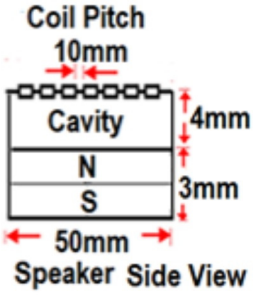

b)

Fig. 6. a) Proposed planar loudspeakers for coils of spiral-in shape plated with copper and aluminum, b) Structure and parameters

The time-domain responses of the sound pressures for the three kinds of loudspeakers using the previously mentioned audio test signal are obtained as shown in Fig. 7(a). The sound pressure levels for the frequencies from $20 \mathrm{~Hz}$ to $20 \mathrm{kHz}$ are also shown in Fig. 7(b), note that the performances of the proposed planar-type loudspeaker for the coil plated with copper are better with lower resistance, and even compatible to that of the traditional loudspeaker. Besides, the proposed loudspeaker has the advantages over the traditional cone-type speaker, e.g. its thickness, weight, structure, cost, manufacturability and versatility. Thus, it can be as a wearable loudspeaker.

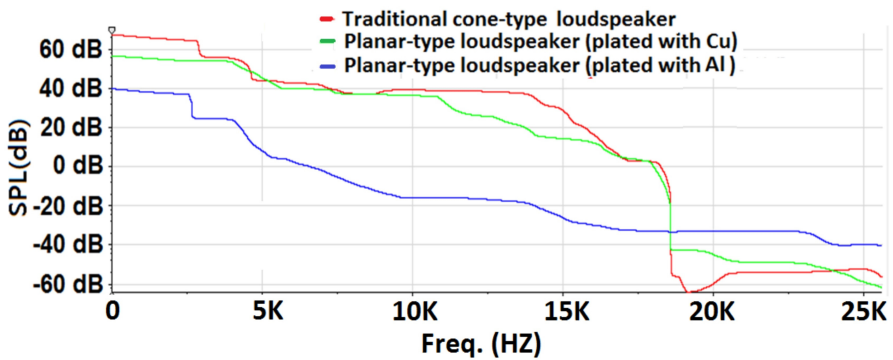

a)

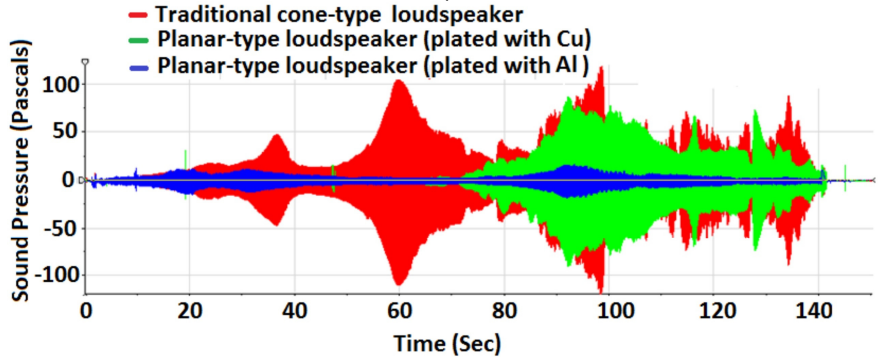

b)

Fig. 7. Performances of loudspeakers with audio test signal for frequencies of $20 \mathrm{~Hz}-20 \mathrm{kHz}$ over $140 \mathrm{~s}$ :

a) time-domain responses of sound pressures in $\mathrm{Pa}$ (Pascals), b) sound pressure levels in $\mathrm{dB}$

\subsection{Aluminum coils with spiral-in, zigzag, and spiral-in and spiral-out shapes}

The time-domain responses of the sound pressures for the three kinds of shapes, e.g. spiral-in, zigzag, and spiral-in and spiral-out, are obtained as shown in Fig. 8(a). The sound pressure levels 
are also shown in Fig. 8(b), one can see the performances of the coil with the shape of spiral-in is better at lower frequencies below $5 \mathrm{kHz}$, while the performance of the zigzag shape is better at middle frequencies between $5 \mathrm{kHz}$ and $15 \mathrm{kHz}$. The performance of the spiral-in and spiral-out one is the worst, because the magnetic flux will be reduced due to the cancellation effect produced by the spiral-in and spiral-out coils, its performance is better only for a small band of higher frequency, e.g. between $15 \mathrm{kHz}$ and $18 \mathrm{kHz}$.
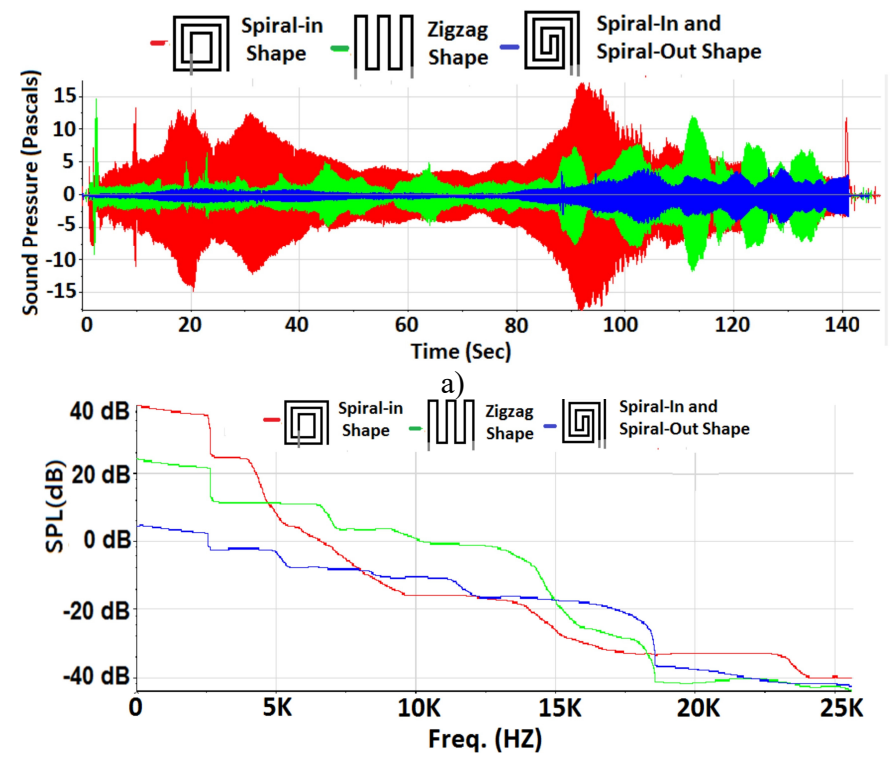

b)

Fig. 8. Performances of aluminum coils with spiral-in, zigzag, and spiral-in and spiral-out shapes:

a) time-domain responses of sound pressures (in $\mathrm{Pa}$ ), b) sound pressure levels (in $\mathrm{dB}$ )

\subsection{Planar rectangular coils with different dimensions and number of turns}

In this section, for easy of comparison three simple acoustical sound enclosures in rectangular boxes as shown in Fig. 9 are applied to compare the SPLs of three rectangular coils with different sizes and number of turns. The dimensions and number of turns of the coils are listed in Table 1 for reference. The curves of SPLs are shown in Figs. 10-12. Note that the smaller the size (\#6), the better the higher frequency response; and the larger the size (\#8), the better the lower frequency response. Thus, the result is reasonable.

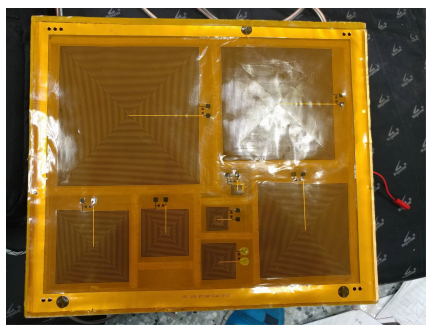

Fig. 9. Several rectangular coils equipped with sound enclosures in rectangular boxes also

Table 1. Three rectangular coils with different dimensions and number of turns

\begin{tabular}{|c|c|c|c|}
\hline Number & $\# 6$ & $\# 7$ & $\# 8$ \\
\hline Dimensions $\left(\mathrm{cm}^{2}\right)$ & $10.15 \times 9.2$ & $12.3 \times 11.6$ & $14.2 \times 13.4$ \\
\hline Number of turns & 41 & 51 & 61 \\
\hline
\end{tabular}




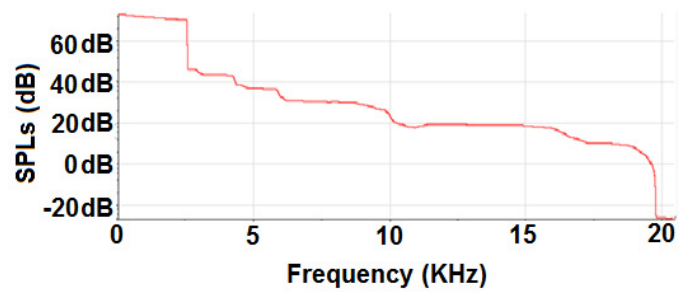

Fig. 10. SPL curve for EFPL \#6

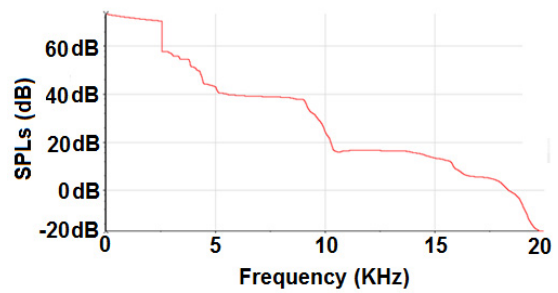

Fig. 11. SPL curve for EFPL \#7

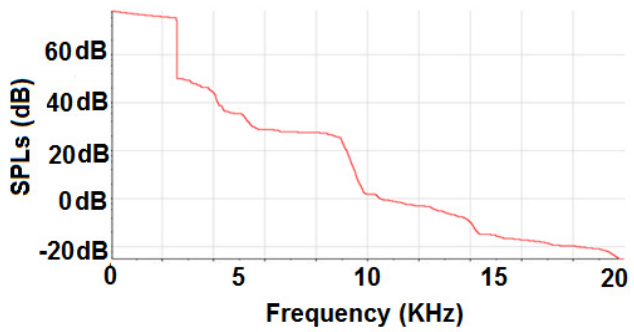

Fig. 12. SPL curve for EFPL \#8

\section{Conclusions}

This paper proposed a novel flexible electro-dynamic planar loudspeaker with coils on polyimide diaphragm that can generate a good audible sound. All the thickness (height) of the cavity, magnet polarization, and diaphragm dimension are taken into consideration. In this study several kinds of planar-type loudspeakers for the coils wounded in spiral-in, spiral-in and spiral-out, and zigzag shapes and plated with copper and aluminum are applied for performance tests, and the comparisons with the traditional cone-type loudspeaker are also made. The proposed loudspeaker has advantages over the traditional cone-type speaker, e.g. its flexibility, simple structure, cost effectiveness, easy manufacturability and application versatility. The idea behind this study using a planar-type loudspeaker with wounded coil on a diaphragm is a novel one, and which was unexplored before.

\section{References}

[1] Suzuki M., Tsuzuki T., KomiyamaT., Yamaguchi T., Furukawa T., Tokito S. Flexible colour OLED display based on phosphorescent material fabricated by ink-jet printing. 13th IDW, 2006.

[2] Fujisaki Y., Sato H., Yamamoto T., Fujikake H., Tokito S., Kurita T. Flexible color LCD panel driven by low-voltage-operation organic TFT. Journal of the Society for Information Display, Vol. 15, 2007, p. 501-506.

[3] Takehiro S., Kazuho O., Akio A., Kohichi K., Akira H., Yuichi M., Akito M. PVDF-driven flexible and transparent loudspeaker. Applied Acoustics, Vol. 70, 2009, p. 1021-1028.

[4] Rashedin R., Meydan T., Borza F. Electromagnetic micro-actuator array for loudspeaker application. Sensors and Actuators, Vol. 129, 2006, p. 118-120.

[5] Lee C. S., Kim J. Y., Lee D. E., Joo J., Wagh B. G., Han S., Beag Y. W., Koh S. K. Flexible and transparent organic film speaker by using highly conducting PEDOT/PSS as electrode. Synthetic Metals, Vol. 139, 2003, p. 457-461.

[6] Arved C. H., Maxi B., Georg C. S., Stefan Z., Andre G., Christian H. Fully mass printed loudspeakers on paper. Organic Electronics, Vol. 13, 2012, p. 2290-2295.

[7] Lin J. M. Eletro-Acoustic Transducer and Method of Manufacturing the Same. U.S. Patent 2013/0163807 A1, 2013.

[8] Fujifilm, http://www.diginfo.tv/v/13-0009-r-en.php.

[9] Yamaha Develops Directional, Flat Panel Speakers - Video Inside. INAVATE, 2010, http://www.inavateonthenet.net. 
[10] SoundPad 580. Warwick Audio Technologies, http://www.warwickaudiotech.com/sites/ default/files/downloads.

[11] Anthony C. ITRI paper-thin flexible loudspeaker won Wall Street journal's technology innovation awards. PRLog - Global Press Release Distribution, 2009, http://prlog.org/10365388.

[12] Zhao Z. Planar Speaker System. US Patent 2013/0243238 A1, 2013.

[13] Je S. S., Junseok C. An electromagnetically actuated micromachined loudspeaker for hearing aids applications. Sensors, Atlanta, 2007.

[14] Graebener D. Single End Planar Magnetic Speaker. U.S. Patent 7251342 B2, July 31, 2007.

[15] Lemarquand G., Ravaud R., Shahosseini I., Lemarquand V., Moulin J., Lefeuvre E. MEMS electrodynamic loudspeakers for mobile phones. Applied Acoustics, Vol. 73, 2012, p. 379-385.

[16] Shahosseini I., Lefeuvre E., Moulin J., Martincic E., Woytasik M., Pillonnet G., Lemarquand G. Planar microcoil optimization of MEMS electrodynamic microspeakers. IEEE Transaction Magnetics, Vol. 49, 2013, p. 4843-4850.

[17] Liou C. H., Chen M. D. Flexible Speaker. U.S. Patent 20090060249 A1, 2009.

[18] Kontomichos F., Koutsioubas A., Mourjopoulos J., Spiliopoulos N., Vradis A. A thermoacoustic device for sound reproduction. Acoustics, Paris, 2008.

[19] Xiao L., Chen Z., Feng C., Liang L., Bai Z. Q., Wang Y., Qian L., Zhang Y., Li Q., Jiang K., Fan S. Flexible, stretchable, transparent carbon nanotube thin film loudspeakers. Nano Letters, Vol. 8, 2008, p. 4539-4545.

[20] Mat D. Electronic Design. 13 December, 2009, http://electronicdesign.com/boards/thin-speakertechnology.

[21] Arnold Magnetic Technologies Corp., "FLEXMAG", Arnold Magnetic Technologies, 2014, http://www.arnoldmagnetics.com.

[22] Beranek L. L., Mellow T. J. Electrodynamic Loudspeakers. Acoustics: Sound Fields and Transducers. Academic Press, 2012, p. 241-288.

[23] Loudspeaker Driver. COMSOL Muitiphysics, 2013, https://www.comsol.com/model/ download/39132/loudspeaker driver.

[24] Daniel R. R. The Science and Applications of Acoustics. Second Edition, Springer Science Business Media Inc., 2006, p. 583.

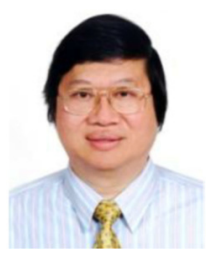

Jium-Ming Lin was born in Taiwan 1952 and graduated from the Department of Electronic Engineering, National Chiao-Tung University at Taiwan in 1974. He achieved the Master and Ph. D Degrees from the same school of Institute of Electronics in 1976 and 1985, respectively. He was an adjunct professor and full professor since 1992 and 1996 at the Dept. of Mechanical Engineering, Chung-Hua University, Taiwan. Now he is a distinguished Professor at Dept. of Electronic Engineering and majors in the fields of planar speaker, RFID, accelerometer, rate gyro; avionics, and wireless measurement technique for health care. He also has several patents in the related areas.

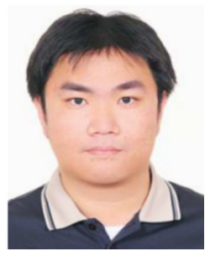

Cheng-Hung Lin was born in Taipei, Taiwan in 1985. Mr. Lin was graduated from the Department of Mechanical Engineering, Chung-Hua University at Hsin-Chu, Taiwan in 2009. He had also achieved the Master Degree from the same school in 2012. His major field was in navigation, guidance and control. His The other interests are as include loudspeaker, RFID, wireless accelerometer and rate gyro, avionics, and MEMS devices. $\mathrm{He}$ is currently a Ph.D. candidate of the doctoral program in engineering science, College of Engineering, at Chung-Hua University. 